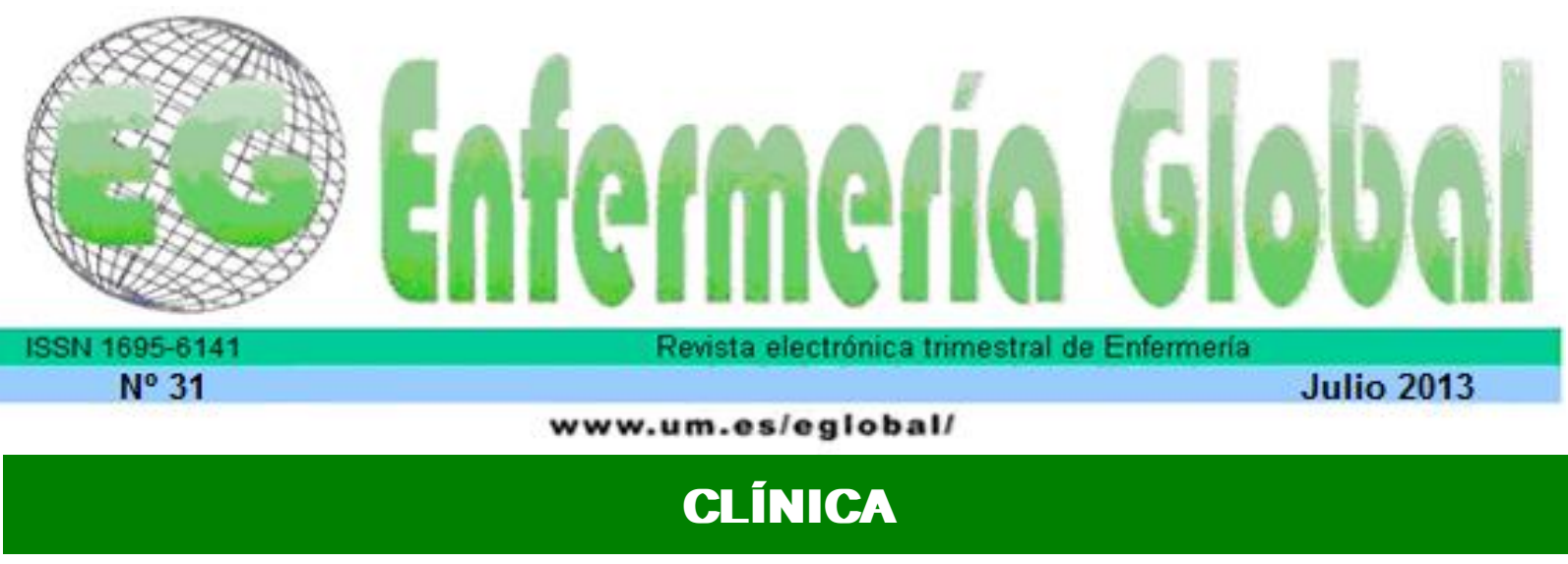

\title{
Valoración del Patrón de la Sexualidad: atención integral de la salud de las personas
}

Valuation of the pattern of sexuality: comprehensive attention of the people's health

\begin{abstract}
*Navarro Sarabia, Julia Isabel **Rodríguez López, Carolina $\mathrm{M}^{\mathrm{a}}$, ${ }^{* * *}$ Garrido Fernández, Pablo **de la Rosa Herrera, Encarnación **Moreno Martínez, Antonia **Robles López, Antonia
\end{abstract}

*Master en Ciencias de la Enfermería. Enfermera de la Unidad de Neumología del Complejo Hospitalario Torrecárdenas de Almería. Profesora Asociada del Departamento de Enfermería de la Universidad de Almería. E-mail: julnasa@hotmail.com **Enfermera de la Unidad de Neumología del Complejo Hospitalario Torrecárdenas de Almería. ***Metodólogo Unidad Apoyo Investigación FIBAO, Fundación para la Investigación Biosanitaria Andalucía Oriental. Complejo Hospitalario Torrecárdenas de Almería.

Palabras clave: Patrones Funcionales; Patrón de la sexualidad y reproducción; sexualidad y salud; Enfermedades de Transmisión Sexual; maltrato; conductas sexuales de riesgo.

Keywords: Functional Patterns; Pattern of sexuality and reproduction; sexuality and health; sexually transmitted diseases; abuse; risky sexual behaviors.

\section{RESUMEN}

Antecedentes: La sexualidad es un componente importante de la vida y la salud. Puede ser origen de graves conflictos relacionados con la salud física, psicológica, incluyendo la transmisión de enfermedades, las experiencias negativas, las pautas sexuales, la violencia y los determinantes culturales o religiosos. Sin embargo, la información recogida en los Registros Enfermeros con frecuencia se limita a la reproducción y / o el estado civil de los pacientes.

Objetivo: El objetivo de este estudio fue evaluar la calidad de la información sobre aspectos relacionados con la sexualidad, los factores que la determinan y la relevancia que los datos obtenidos puedan tener sobre la salud de las personas.

Métodos: Estudio Observacional realizado en la Unidad de Neumología de un Hospital de referencia provincial en el que se revisaron 250 Valoraciones Iniciales (VI) Historias Clínicas y se entrevistó de forma consecutiva a 292 pacientes ingresados mediante cuestionario. En una primera etapa, se evaluó la información recogida y la naturaleza de los datos obtenidos acerca de la información relacionada con la sexualidad en $250 \mathrm{VI}$ de las Historias Clínicas de pacientes ingresados el año anterior a la realización del estudio. En un segundo paso, se elaboró un cuestionario, basado en los criterios de 
valoración de los Patrones Funcionales de Gordon y centrado en la valoración del Patrón de la sexualidad y se aplicó a 292 pacientes hospitalizados. Se investigó la influencia los diferentes aspectos de este patrón mediante el empleo de la prueba de Chi-cuadrado.

Resultados: De las $250 \mathrm{VI}$ que se revisaron, se constató que en la VI se registraban los datos de reproducción en el $75 \%$ de las VI. En la segunda fase, se incluyeron 292 sujetos, $46 ., 9 \%$ hombres y $53,1 \%$ mujeres, con edad media de 57 años, y la tasa de respuesta al cuestionario en los pacientes fue del $100 \%$, siendo el porcentaje de respuesta superior al $95 \%$ en todas las preguntas.

Se recogió: revisiones por el especialista, utilización de métodos conceptivos, padecimiento de ETS, calidad de relación con la pareja, percepción del sentimiento de su pareja, importancia de las relaciones sexuales en su vida, antecedentes de violencia sexual, relaciones fuera de pareja estable.

En el desarrollo del trabajo se identificaron Diagnósticos Enfermeros relevantes para la evolución de la salud integral de las personas en el $70,2 \%$ de los casos.

Conclusiones: El porcentaje de respuesta a las preguntas del cuestionario fue elevado. Las diferencias en el Patrón de la sexualidad influyeron en la mayoría de las condiciones que requieren la salud integral de las personas. La información recogida tuvo relevancia para la salud integral de las personas en un número elevado de casos.

La calidad de la información recogida fue buena, ya que las personas informaron sin reserva y naturalidad, como del resto de los patrones a las preguntas formuladas sobre su sexualidad. Se identifican problemas de prácticas de riesgo y maltrato en un número elevado de personas, que indican la necesidad de su valoración. No preguntar sobre este Patrón o hacerlo solo referido a la reproducción, hace que quede sesgada la información al respecto, en muchas ocasiones con serias repercusiones sobre la salud de las personas.

Las enfermeras tienen competencias sobre la salud sexual de las personas y han de ser sus conocimientos científicos las que determinen la idoneidad de la toma de decisiones a este respecto.

\section{ABSTRACT}

Background: Sexuality is an important component of life and health. Also of severe conflicts related to physical or psychological events, including the transmission of diseases, negative experiences, sexual patterns, violence and cultural or religious determinants. Nevertheless, the information collected in the nurse's files is frequently limited to the reproductive and/or marital status of the patients.

Objective: The objective of this study was to evaluate the quality of information on issues related to sexuality, its determinants and the iof the data obtained can have on people's health

Methods: Observational study conducted in the respiratory medicine department of a provincial referral hospital where initial assessments (IA) were revised 250 medical charts and interviewed 292 consecutively admitted patients by questionnaire focused.

In a first step, the information collected and the nature of the data about the information related to sexuality in 250 IA of medical records of patients admitted in the year before the study.

In a second step, a questionnaire was developed, based on the evaluation criteria of functional patterns Gordon and focused on assessing the pattern of sexuality and applied to 292 patients hospitalized. The influence of different aspects of this pattern using the Chi-square test.

Results: In the first step of the 250 IA reviewed, it was found that in the IA data were recorded breeding in $75 \%$ of the IA.

In the second phase, 292 subjects were included, $46.9 \%$ men and $53.1 \%$ women, mean age 57 years, and the response rate to the questionnaire in patients was $100 \%$, with a higher response rate $95 \%$ in all questions. 
Was collected: reviews by the specialist, use of contraceptives methods, STD condition, quality of relationship with partner, perceived sense of your partner, the importance of sex in his life, a history of sexual violence, stable partner relationships outside.

In the development labor Diagnoses were identified relevant to the evolution of the overall health of people in $70.2 \%$ of cases.

Conclusions: The response rate to the questionnaire was high. The differences in the pattern of sexuality influenced most health conditions requiring full of people. The information collected was relevant to the overall health of people in a large number of cases.

Problems are identified risk practices and abuse in a large number of people, indicating the need for assessment. Do not ask about this pattern or do only referred to the reproduction, it is biased for the information, often with serious implications for the health of people.

Nurses have expertise on sexual health of people and should be the scientific knowledge to determine the suitability of the decision making in this regard.

\section{INTRODUCCIÓN}

La Organización Mundial de la Salud (OMS) define la salud sexual como la integración de los elementos somáticos, emocionales, intelectuales y sociales del ser sexual, por medio que sean positivamente enriquecedores y que potencien la personalidad, la comunicación y el amor ${ }^{1}$. El patrón de la sexualidad se refiere al conjunto de estrategias, conductas y actitudes que se utilizan en la búsqueda del placer sexual, y describe tanto los patrones de satisfacción o insatisfacción de la sexualidad como el patrón reproductivo ${ }^{2}$.

El comportamiento humano alrededor de la reproducción y el placer sexual ha sido tabú para todas las culturas y el hecho de establecer normas de conducta apropiadas y universales en lo que se refiere a la vida sexual es tarea difícil. Los factores socioculturales en cuanto al nivel de información al que se tiene acceso sobre el tema, no han evolucionado paralelamente. Además de las conductas y actitudes humanas elementales en el ciclo vital, es preciso mostrar la interrelación que tienen las connotaciones transculturales sin obviar las biológicas y psicológicas. En ocasiones, se presentan disfunciones de tipo sexual, que en la mayoría de los casos tiene un origen psicológico, mientras que otras son debidas a desordenes fisiológicos.

Las enfermeras/os disponen de herramientas como los Diagnósticos de Enfermería de la North American Nursing (NANDA), que están relacionados con alteraciones en la sexualidad de las personas y que mediante la identificación de los mismos pueden colaborar a prevenir y mejorar la salud.

Por otro lado, las enfermedades de transmisión sexual (ETS) son un problema de salud. El Síndrome de la Inmunodeficiencia humana, (SIDA) es una enfermedad hoy día de transmisión fundamentalmente sexual, y cualquier individuo activo sexualmente tiene riesgo de contraerla. ${ }^{3-9}$

En la Unidad donde desarrollamos nuestra práctica asistencial, hemos observado que algunos pacientes con un patrón de la sexualidad no común no evolucionaron satisfactoriamente. Tal es el caso de una paciente de 22 años ingresada previamente en cinco ocasiones, dos de ellas por intento de autolisis en Salud Mental. Al preguntar si sus relaciones sexuales eran o no satisfactorias, informó que desde que tenía 8 
años había sido violada reiteradamente por la pareja de su madre. Sin embargo, este hecho no estaba reflejado en ningún episodio de sus múltiples ingresos anteriores. Otro paciente de 32 años ingresó por neumonía, produciéndose un desenlace fatal en 48 horas. Realizada la autopsia, se detectó la infección por el Virus de la Inmunodeficiencia Humana (VIH), siendo ésta la causa de su mala evolución. En este caso tampoco se le había preguntado sobre relaciones de riesgo, y posteriormente se confirmó que fue a través del sexo como se produjo el contagio.

Estos acontecimientos nos motivaron a realizar el presente estudio. El objetivo principal de este trabajo es evaluar la calidad de la información sobre aspectos relacionados con la sexualidad, los factores que la determinan y la relevancia que los datos obtenidos puedan tener sobre la salud de las personas. El objetivo secundario es evaluar si existen diferencias por sexo respecto a las alteraciones identificadas en las personas evaluadas.

\section{METODOLOGÍA}

Estudio Observacional descriptivo transversal, realizado en la Unidad de Neumología del Complejo Hospitalario Torrecárdenas de Almería, desde Marzo de 2009 hasta Marzo de 2010. Teniendo en cuenta la información que el Servicio de Informática nos proporcionó respecto al número de ingresos que se habían producido durante el año 2008 en la Unidad de Neumología, se calculó un tamaño muestral de 292 pacientes, con un error máximo aceptable menor de un $5 \%$, con un límite de confianza de un 95\% y una proporción de la población máxima estimada de 0,5.

Se incluyeron 292 pacientes ingresados en la Unidad de Neumología, de forma secuencial consecutiva. Se tuvieron en cuenta los siguientes criterios de exclusión: personas menores de 18 años y mayores de 85 años, pacientes con alteraciones del patrón cognitivo (demencias, alteraciones de la conciencia...) y personas ingresadas por violación.

En una primera fase del trabajo, se revisó la información recogida en 250 Valoraciones Iniciales (VI) de pacientes ingresados en la Unidad de Neumología durante el año 2009, utilizando para la evaluación de la cantidad y calidad de la información recogida los criterios estandarizados de valoración de los Patrones funcionales de Gordon ${ }^{10}$, constatando que en la VI se registraba el estado civil, el número de hijos y si estos continúan viviendo o no con la pareja, y datos referidos a la reproducción.

En la segunda parte del estudio, los datos se obtuvieron durante la entrevista de la VI que se realiza al ingreso de los pacientes, utilizando el formato oficial del hospital, siguiendo un cuestionario modificado de los criterios de valoración de los Patrones Funcionales de M. Gordon del Observatorio de enfermera ${ }^{10}$ para realizar la entrevista y valoración del Patrón de la Sexualidad, con la finalidad de homogeneizar las preguntas realizadas a los pacientes por las 5 enfermeras de la Unidad que participaron en el trabajo (Anexo 1).

Previamente se les informó a los pacientes de la confidencialidad de los datos, así como de la libertad para no responder a aquellas preguntas de la VI. que no deseasen. ${ }^{11}$ Se realizó hoja de informe del estudio y confidencialidad, para la información y solicitud de consentimiento de los personas objeto de estudio, y se obtuvo el permiso de la Subcomisión de Investigación del Complejo Hospitalario Torrecardenas. 


\section{Análisis estadístico.}

Los datos se procesaron mediante el uso del paquete estadístico SPSS versión 17.0. Las fases de análisis estadístico son las siguientes:

Fase 1: Estadística descriptiva mediante cálculo y representación de los valores centrales (media) y dispersión (desviación estándar), y de tablas porcentuales.

Fase 2: Análisis bivariante, se compararon por sexo los diferentes ítems mediante la prueba Chi-cuadrado de Pearson con corrección por continuidad de Yates y la prueba exacta de Fisher, considerando significativos para todas las pruebas $\mathrm{p}$ valores inferiores a 0,05.

\section{RESULTADOS Y DISCUSIÓN}

De las $250 \mathrm{VI}$ que se revisaron en la primera fase del estudio, se constató que en la VI se registraba el estado civil, el número de hijos y si estos continúan viviendo o no con la pareja en el $75 \%$ de las VI. No se constató la recogida de otro tipo de información.

En la segunda fase del estudio, se incluyeron 292 sujetos, $137(46,9 \%)$ hombres y 155 mujeres (53,1\%), con edad media de 57 años con una desviación típica de 16,46. El $62 \%$ estaban casados, el $10 \%$ solteros, el $14 \%$ eran viudos, el $7 \%$ vivían en pareja y el $8 \%$ estaban separados.

El motivo de ingreso fue: Enfermedad Pulmonar Obstructiva Crónica $(32,9 \%)$, neumonía (13\%), asma (12,3\%), tuberculosis $(11,3 \%)$, derrame pleural $(10,3 \%)$, cáncer de pulmón $(6,8 \%)$, tromboembolismo pulmonar (5,5\%), fibrosis pulmonar $(5,1 \%)$, hemoptisis $(2,4 \%)$, atelectasia $(0,3 \%)$.

La tasa de respuesta al cuestionario en los pacientes fue del 100\%. La mayoría de los pacientes respondió a todas las preguntas del cuestionario, con una rango de respuesta entre el $95,2 \%$ y el $100 \%$.

\begin{tabular}{|cl|}
\hline Preguntas & $\begin{array}{l}\text { Porcentaje de } \\
\text { Respuestas ( } \boldsymbol{n = 2 9 2 )}\end{array}$ \\
\hline Acude a revisiones Ginecólogo- Urólogo & $100 \%$ \\
Revisiones prevención del Cáncer & $99,3 \%$ \\
Estado civil & $100 \%$ \\
Número de hijos & $100 \%$ \\
Número de abortos & $100 \%$ \\
Métodos anticonceptivos que utiliza o ha utilizado & $100 \%$ \\
Quien se los ha recomendado prescrito & $98,3 \%$ \\
Fecha menarquia, fecha menopausia & $100 \%$ \\
Síntomas post-menopausia & $100 \%$ \\
\hline Que hace para paliar los síntomas post- & $100 \%$ \\
menopausia & \\
\hline Ha padecido alguna enfermedad de transmisión & $97,9 \%$ \\
sexual (ETS) & \\
\hline Como considera que son sus relaciones con su & $96,2 \%$ (no contestaron \\
pareja & los que no tenían \\
& pareja) \\
\hline
\end{tabular}




\begin{tabular}{|c|c|}
\hline Se siente querido por su pareja & 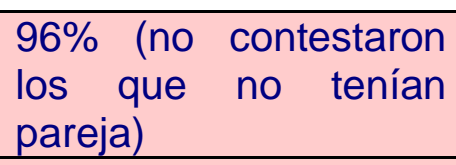 \\
\hline Se siente reconocido por su pareja & $\begin{array}{l}95,2 \% \text { (no contestaron } \\
\text { los que no tenían } \\
\text { pareja) }\end{array}$ \\
\hline $\begin{array}{l}\text { Como considera que son sus relaciones sexuales } \\
\text { con su pareja }\end{array}$ & $\begin{array}{l}96,2 \% \text { (no contestaron } \\
\text { los que no tenían } \\
\text { pareja) }\end{array}$ \\
\hline $\begin{array}{l}\text { Las relaciones sexuales son importantes para su } \\
\text { vida }\end{array}$ & $100 \%$ \\
\hline Ha disminuido su interés sexual & $99,3 \%$ \\
\hline $\begin{array}{c}\text { Piensa que su enfermedad influye en la cantidad } \\
\text { o calidad de sus relaciones }\end{array}$ & $100 \%$ \\
\hline La disminución de su interés sexual & $100 \%$ \\
\hline $\begin{array}{l}\text { Considera que las relaciones sexuales son igual } \\
\text { de importantes para el hombre que para la mujer }\end{array}$ & $100 \%$ \\
\hline Violencia en sus relaciones & $99,7 \%$ \\
\hline Tipo de violencia (física/psíquica) & $100 \%$ \\
\hline Violencia por parte de su pareja & $99 \%$ \\
\hline Por otros & $99 \%$ \\
\hline Abusos sexuales & $99,3 \%$ \\
\hline Abusos en edad infantil & $99,3 \%$ \\
\hline Abusos en edad adulta & $99,3 \%$ \\
\hline Por su pareja & $99,3 \%$ \\
\hline Por familiares/conocidos & $99,3 \%$ \\
\hline Por desconocidos & $99 \%$ \\
\hline $\begin{array}{l}\text { Relaciones con personas que no fuesen pareja } \\
\text { estable }\end{array}$ & $98,6 \%$ \\
\hline $\begin{array}{l}\text { Relaciones esporádicas con personas } \\
\text { desconocidas }\end{array}$ & $99 \%$ \\
\hline Relaciones con personas del mismo sexo & $97,3 \%$ \\
\hline Relaciones con personas de ambos sexos & $95,9 \%$ \\
\hline Relaciones con personas de distintos sexos & $97 \%$ \\
\hline $\begin{array}{c}\text { En caso de haber tenido relaciones con } \\
\text { desconocidos que medidas de barrera ha } \\
\text { utilizado }\end{array}$ & $100 \%$ \\
\hline
\end{tabular}

Un total de $117(86,6 \%)$ hombres no acudían a revisiones por urólogo mientras que 88 $(56,8 \%)$ mujeres no realizaban revisiones ginecológicas de forma habitual. Las mujeres tenían una razón de ventaja de realizar revisiones respecto a los hombre de 4,6 con un intervalo de confianza (IC) del 95\% $(2,63 ; 8,37)$.

Admitieron no utilizar ningún tipo de medida anticonceptiva $30(22,7 \%)$ hombres y 19 $(12,3 \%)$ mujeres. Los hombres tenían una probabilidad de no utilizar métodos anticonceptivos 2,1 veces mayor que las mujeres con un IC 95\% $(1,12 ; 3,95)$.

Respecto a quién le había recomendado el método anticonceptivo, $41,8 \%$ de la población informó que había sido por iniciativa propia, 37,4\% un familiar o amigo, $16,4 \%$ el médico, $4,5 \%$ el farmacéutico, y $0,7 \%$ la enfermera. 
Respecto a las medidas de barrera, $81(65,3 \%)$ personas no habían utilizado ninguna cuando mantuvieron relaciones con personas desconocidas. La probabilidad de que los hombres no utilicen métodos de barrera es mayor que la de las mujeres, con una OR de 4,8 y un IC del $95 \%(1,8 ; 12,7)$ [figura 1]

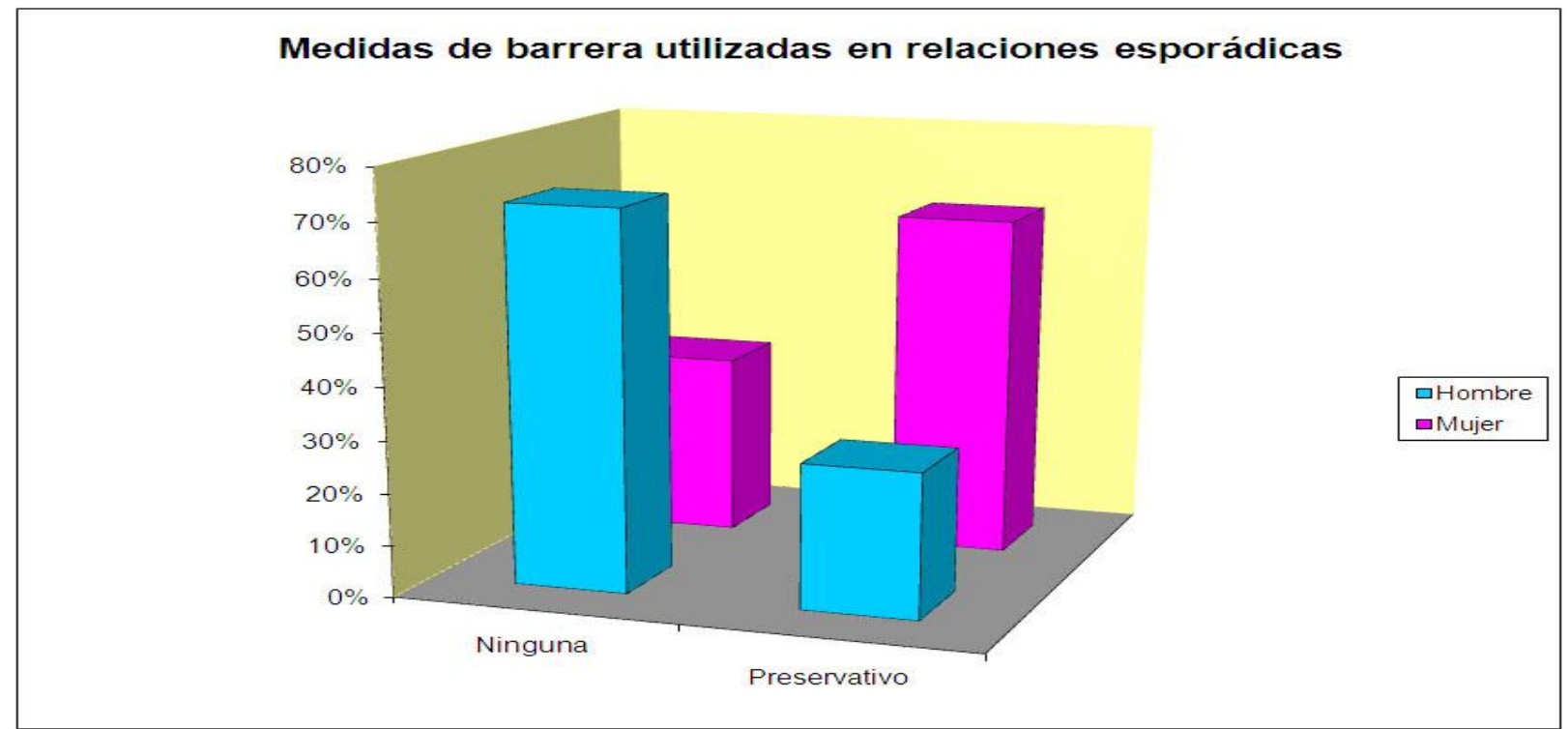

Figura 1

El $82,3 \%$ de las mujeres que estaban en la etapa de menopausia informó que no había hecho nada para paliar los síntomas que padecía relacionados con ésta. Un $4,2 \%$ de las mujeres utilizaba lubricantes para mejorar sus relaciones sexuales, el $12,5 \%$ consultó al médico para solucionar sus problemas, y el $1 \%$ consultó a la enfermera/o.

Un 22\% informó haber padecido ETS. Seis personas no respondieron a este respecto, lo que supuso un porcentaje de pérdidas del $2,1 \%$. Un total de 53 hombres $(39,3 \%)$ y 10 mujeres $(6,6 \%)$ habían padecido alguna ETS, siendo el riesgo 9 veces mayor en los hombres respecto a las mujeres, con un IC $95 \%(4,4 ; 18,9)$

Respecto a la información de cómo percibían sus relaciones de pareja, $79(56,8 \%)$ de hombres informaron que eran buenas comparado con 50 (33,6\%) mujeres; $p<0,001$ [figura 2]. 


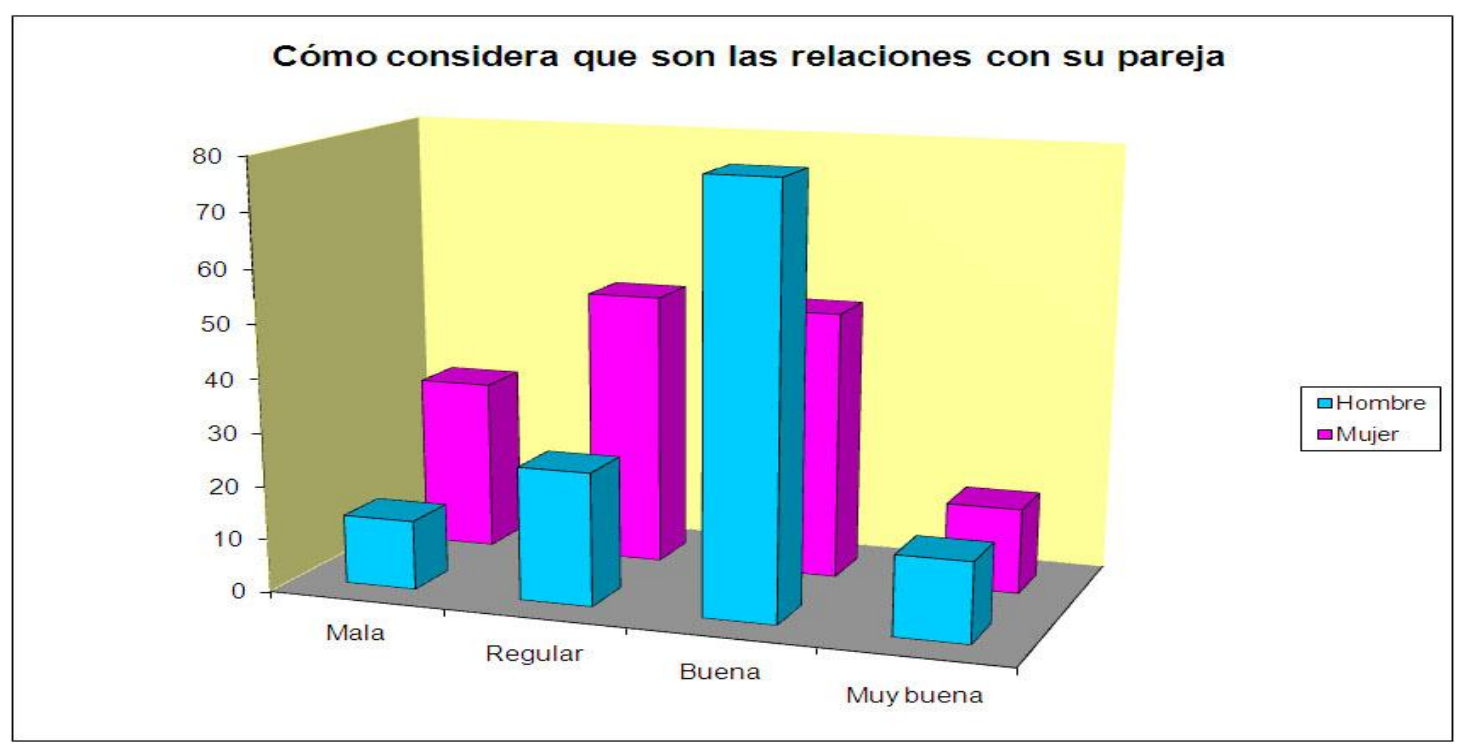

Figura 2

El $23,2 \%$ de la población no se sentía querido por su pareja, 17 (13\%) hombres comparado con 48 (32,2\% mujeres que afirmaron sentirse queridos por sus parejas; $\mathrm{p}<0,001$.

Respecto a si sus relaciones sexuales son satisfactorias, $113(40,2 \%)$ personas las definen como satisfactorias, $95(33,8 \%)$ personas poco satisfactorias, $58(20,6 \%)$ personas nada satisfactorias, $15(5,3 \%)$ personas muy satisfactorias, $11(3,8 \%)$ personas no respondieron a esta pregunta. [Figura 3]

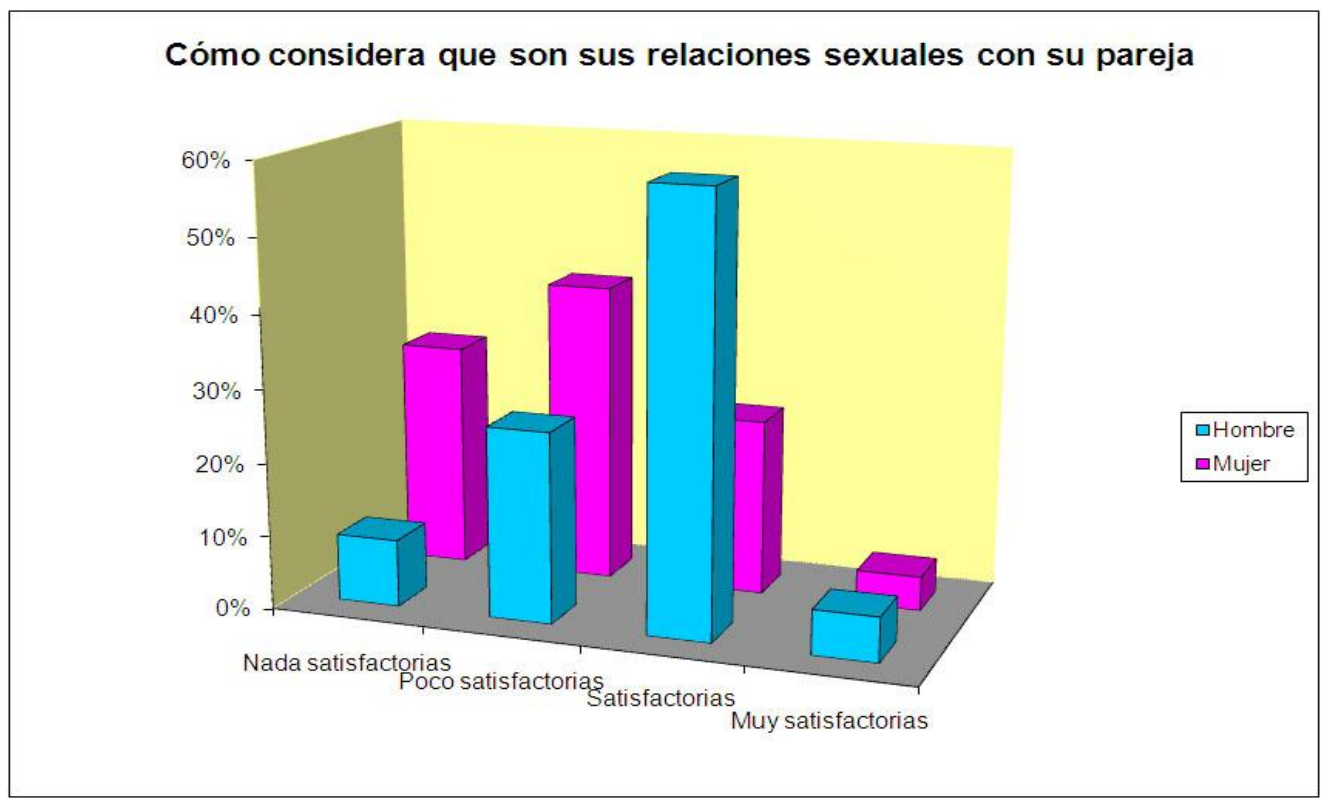

Figura 3

Respecto a la importancia que tienen las relaciones sexuales en su vida: $23(16,8 \%)$ hombres y $86(55,8 \%)$ mujeres respondieron que no son importantes, por lo que 109 $(37,5 \%)$ individuos sin distinción de sexo no consideran importantes las relaciones sexuales para su vida. Sí son importantes para su vida para $114(83,2 \%)$ hombres y $68(44,2 \%)$ mujeres, siendo esta diferencia ente ambos sexos estadísticamente significativa, con una odds ratio para sexo mujer/hombre de 0,16 con un IC $95 \%$ 
$(0,09 ; 0,28)$.

Un mayor porcentaje de mujeres reportó haber padecido violencia en las relaciones sexuales comparado con los hombres $(40.0 \%$ vs $21,3 \%$; $p<0,001$, respectivamente). Solo 3 casos fueron denunciados por mujeres. Es 2,46 veces más probable que los hombres no padezcan violencia en sus relaciones.

Un 15,1\% informó haber padecido violencia física y el 14,4\% psíquica [Tabla I]

Tabla I
\begin{tabular}{lll} 
& & \\
Tipo de violencia & & \\
\hline Física & 44 & 15,1 \\
\hline Psíquica & 42 & 14,4 \\
No violencia & 206 & 70,5 \\
Total & 292 & 100
\end{tabular}

Un total de $168(58,3 \%)$ personas afirmaron no haber tenido relaciones fuera de pareja estable, mientras que $120(40 \%)$ personas si las habían tenido y $4(1,7 \%)$ personas, no respondieron a esta pregunta $(p<0,01)$. 81 hombres y 12 mujeres informan haber mantenido relaciones con personas desconocidas. Los hombres tienen 17,2 veces más posibilidades de tener relaciones esporádicas con personas desconocidas que las mujeres con un IC del $95 \%(8,77 ; 34,48)$ [figura 4].

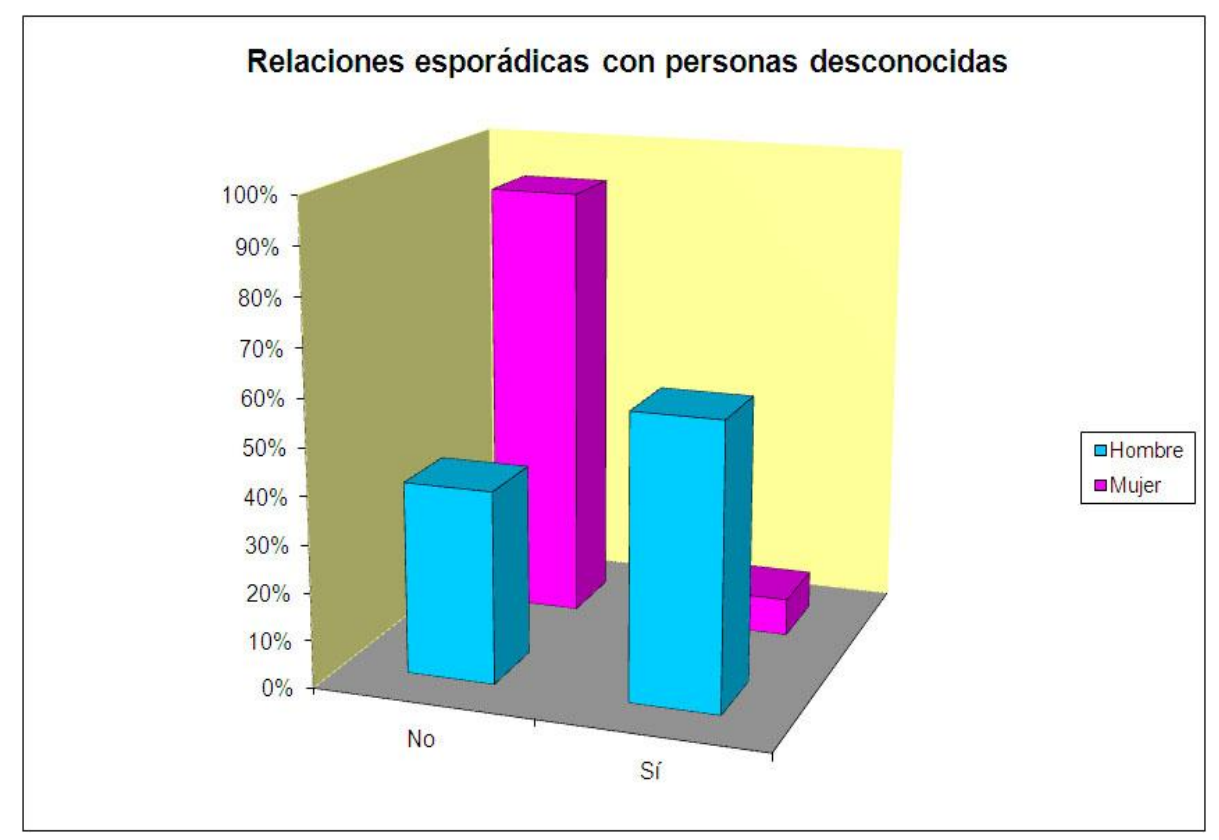

figura 4

La recogida sistematizada de datos respecto al Patrón de la sexualidad aportó información que permitió la identificación de los Diagnósticos Enfermeros ${ }^{12-14}$ en el $70,2 \%$ de los pacientes, siendo la frecuencia de estos diagnósticos la siguiente:

- Tendencias a adoptar conductas de riesgo para la salud (00188) $27,7 \%$ 
- Conflicto de decisiones (00083) 81,9\%.

- Mantenimiento ineficaz de la salud (00099) 83,8\%.

- Patrón sexual inefectivo (00065) 43,1\%

- Síndrome traumático de la violación (00142) 20,7\%

- Riesgo de violencia dirigida a otros (00138) $47,6 \%$

- Conocimientos deficientes (00126): Conductas sanitarias, Fomento de salud 91,2\%.

\section{DISCUSIÓN}

A pesar de que se formularon preguntas que pudieran considerarse comprometidas, por corresponder a un área personal e íntima de las personas, el porcentaje de respuesta de las personas a las preguntas en ningún caso fue inferior al $95 \%$. No observándose diferencias relacionadas con el sexo de las personas en dicho porcentaje de respuesta.

La información recogida tuvo relevancia para la salud integral de las personas en un número elevado de casos. La Ley de 28 de Mayo de cohesión y calidad del Sistema Nacional de Salud, en el artículo 2 hace referencia al derecho a una atención sanitaria integral. La profesión enfermera está sujeta a unos deberes regulados en la Ley 44//2003 de 21 de noviembre, de ordenación de las profesiones sanitarias.

Las preguntas que se realizaron relativas a la condición sexual o preferencias no tuvieron otra finalidad sino la de identificar si pudieran ser relaciones de riesgo. En ningún caso entendemos que la condición sexual de una persona haya de ser enjuiciada por las enfermeras. Los datos obtenidos referentes a las revisiones médicas para la prevención y detección de enfermedades como el cáncer, tanto en hombres como en mujeres es relativamente bajo, teniendo en cuenta que se disponen de programas para la detección precoz del cáncer. La diferencia entre hombres y mujeres puede ser justificada en parte, por el hecho de que actualmente están más dirigidos a la prevención en las mujeres, se da más información en medios de comunicación a este respecto.

Las enfermeras de Atención Especializada y de Atención Primaria deben tomar conciencia de la competencia e importancia que tienen en el asesoramiento respecto a la prevención del cáncer tanto en hombres como en mujeres. A. Rodríguez Castro (2011), realiza una revisión de artículos que analizan algunos programas, que incorporan la Educación para la Salud (EpS), impartidos en Atención Primaria, aportándose orientaciones que pueden colaborar en hacer coherente la teoría con la práctica y que puedan contribuir al desarrollo de políticas sanitarias.

Las relaciones fuera de pareja estable igualmente fueron valoradas, tanto si se mantuvieron con o sin las medidas de barrera, por haber tenido en cuenta que puede tener una repercusión directa sobre su salud y la de su pareja. 81 personas habían mantenido relaciones sexuales de riesgo. ${ }^{15-18}$

"Debido al papel que desempeñan las enfermedades de transmisión sexual adicionales, la pareja debe entender la necesidad de definir reglas sobre 
contactos sexuales fuera de esa relación estable" "El condón no debe dejar de utilizarse" porque "la susceptibilidad de cada persona al VIH es distinta", comentó el panameño José Rafael Olmedo, integrante de la Coalición Global de Jóvenes contra el Sida (Agosto 2008).

Los datos recogidos identifican maltrato físico y/o psicológico en 91 personas, 29 hombres y 62 mujeres. Los profesionales de la salud no podemos mantenernos al margen en la violencia de género, el alarmante aumento de muertes de personas por esta causa es competencia también de todos los profesionales de la salud. Según datos del Ministerio de Sanidad, Política Social e Igualdad, en 2010 se realizaron 134.000 denuncias por violencia de género, 76 personas perdieron la vida por esta causa de las cuales 68 eran mujeres.

Los pacientes en ningún caso hicieron alusión a sentirse violentos por las preguntas realizadas. Su participación y porcentaje de respuesta fue alto, no hubo pérdidas en el estudio. Manifestaron estar aliviados por poder hablar de este tema con un profesional y sentirse escuchado. Los profesionales que intervinieron en el estudio estaban sensibilizados, se informaron y formaron previamente. Realizaron la valoración con empatía, discreción y respeto.

Como posibles limitaciones del estudio, destaca la poca intimidad que existe en las habitaciones de los pacientes, para realizar las Valoraciones. Los profesionales de Enfermería en general son reticentes a valorar este patrón, argumentando tanto que es una parcela íntima de las personas, como que sus propios valores y creencias le impiden realizar determinadas preguntas, otras argumentaron no tener conocimientos para realizar las preguntas y aportar soluciones.

Esto se podría considerar, tal vez, tanto una fortaleza como una debilidad, ya que puede propiciar el realizar un estudio cualitativo acerca de las razones que los profesionales de Enfermería tienen y/o argumentos para limitar la información referida a este Patrón. En búsqueda bibliográfica realizada tras la realización del trabajo, no encontramos publicaciones en castellano, referidas a la valoración de este Patrón, ni sobre las actitudes de los profesionales de Enfermería a este respecto.

\section{CONCLUSIONES}

Valorar el Patrón de la sexualidad de forma exhaustiva, evidencia la mejora de la información cuali-cuantitativa, para el cuidado de los pacientes.

La información recogida de este Patrón aumenta la seguridad de los pacientes por las repercusiones que su omisión puede tener sobre la salud de las personas.

Se evidencia una cierta relajación en la utilización de medidas de barrera en las prácticas sexuales, constituyéndose en relaciones de riesgo para las personas. La prevención, la detección precoz y la información sobre el SIDA son competencias de Enfermería.

El maltrato y las violaciones se han detectado en un número elevado de personas.

Según la información estadística de violencia de género - Diciembre de 2010, del Ministerio de Sanidad Política Social e Igualdad-, en ese año se produjeron 73 víctimas mortales. Los profesionales de la Enfermería estamos obligados a asumir las competencias que tenemos a este respecto. 
Los conocimientos científicos, la rigurosidad, la observación, las habilidades, la coherencia y el sentido común, de los profesionales deben ser los principios para evaluar la pertinencia, circunstancias o necesidad de realizar determinadas preguntas, y las personas decidirán si contestan o no.

\section{REFERENCIAS BIBLIOGRÁFICAS}

1. Organización Mundial de la Salud (2006). Defining sexual health: report of a technical consultation on sexual health, 28-31 January 2002, Geneva.

2. Gordon M. Diagnóstico Enfermero, proceso y aplicación. 3aㅡ. Bd. BAC: Mosby/Doyma 1996.

3. Jaramillo N. Sexualidad humana (Internet). Disponible en: http://www.efiro.com/canal/contenido/sexualidad-humana-1012962.php. Consultado 4 de febrero 2.009

4. Marchador Pinillos B, Martínez López M. Sexualidad y adolescencia. Puesta al día sobre publicaciones. Index Enferm. 2003 inv. XII(43):91.

5. Raposo Carrasco, L. Plan de cuidados 67: la sexualidad en la etapa de la menopausia. Comunic Enferm. 2000. 3(15):7-9. Caso clínico.

6. http://www.jonkepa.wordpress.com/2007/07/02/la-sexualidad/ Consultado 25 de Abril 2008.

7. Romero de Castilla Gil, RJ Lora Cerezo, N y Cañete Estrada, R. Adolescentes y fuentes de información de sexualidad: preferencias y utilidad percibida. Aten Primaria. 2001 ene. 27(1):12-17.

8. http://www.mcsweb.org/sida/10_preguntas_sida.pdf (Consultado 26 de Abril 2008).

9. Alfaro, R. Aplicación del Proceso Enfermero. Guía paso a paso. 4 Ed. SpringerVerlang Ibérica. Barcelona. 1999.

10. Observatorio de enfermera. Criterios estandarizados de valoración de los Patrones Funcionales de M. Gordon. Disponible en: http://www.ome.es/04_01_desa.cfm?id=391\#ancla9 (consultado el 16 Octubre de 2009)

11. Ley Orgánica 15/1999, de 13 de diciembre, de Protección de Datos de Carácter Personal. Pub. Boletín Oficial del Estado Num. 298 (14 Dic. 1999).

12. NANDA. Diagnósticos enfermeros: Definiciones y Clasificación 2007-2008: Madrid-Barcelona: Elsevier; 2008.

13. Moorhad Sue, Johnson Marion, Maas Meridean, Clasificación de Resultados de Enfermería (NOC), Tercera Ed. Mosby. Eselvier. Madrid, 2007.

14. McCloskey Dochterman Joanne, Bulechek, Gloria M Clasificación de Intervenciones de Enfermería. Cuarta ed. Eselvier. Madrid. 2007

15. Violencia sexual ITS-VIH-Sida hacia un modelo de atención humanizada. Recuperándose de la violación sexual. Disponible en: http://www.ecapsocial.com.ar/files/Violencia-sexual.pdf (Consultado 8 de Marzo 2011)

16. Medidas para dar respuesta al $\mathrm{VIH}$ y al SIDA. Manual para parlamentarios no 15. Unión parlamentaria, PNUD, ONUSIDA. 2007. Disponible en: http://www.ipu.org/PDF/publications/aids07-s.pdf (Consultado 5 de Febrero 2011)

17. Estrategia de ONUSIDA para 2011- 2015. Disponible en: http://www.unaids.org/es/strategygoalsby2015/ (Consultado 5 Febrero 2011).

18. Rodriguez Zazo. Las 10 preguntas más frecuentes sobre SIDA, disponible en http://www.jarodriguezzazo.net (consultado 15 Abril 2009) 


\section{ANEXO 1}

\section{GUÍA SOPORTE ENTREVISTA}

- Acude a revisiones Ginecólogo - Urólogo Si $\square$ No $\square$

- Revisiones de prevención del cáncer Si $\square$ No $\square$

- Estado civil casado $\square$ soltero $\square$ viudo $\square$ vive en pareja $\square$ separado $\square$

- Número de hijos

- Número de partos

- Abortos Si $\square$ No $\square$

- Edad de la menarquia Edad de la menopausia

- Métodos anticonceptivos que utiliza o ha utilizado vasectomía $\square$ ligadura de trompas $\square$ anticonceptivos $\square$ preservativo $\square$ Coitus interruptus $\square$ castidad $\square$ método Ogino $\square$ Sintotérmico $\square$ Billings $\square$

- Quien se los ha recomendado y/o prescrito farmacéutico $\square$ médico $\square$ enfermera/o $\square$ amiga $\square$ familiar $\square$

- Síntomas post-menopaúsicos: disminución de la líbido $\square$ Sofocos $\square$ depresión $\square$ relaciones dolorosas $\square$ sequedad vaginal $\square$ otros (describir)

- Que hace para paliar los síntomas: nada $\square$ utilizar lubricantes $\square$ consultar médico $\square$ consultar enfermera $\square$

- Ha padecido alguna ETS Si $\square$ No $\square$

- Cómo considera que son las relaciones con su pareja: Mala $\square \quad$ regular $\square$ buena $\square$ muy buena $\square$

- Se siente querido por su pareja: Si $\square$ No $\square$

- Se siente reconocido por su pareja $\mathrm{Si} \square \mathrm{No} \square$

- Cómo considera que son sus relaciones sexuales con su pareja nada satisfactorias $\square$ poco satisfactorias $\square$ satisfactorias $\square$ muy satisfactorias $\square$

- Las relaciones sexuales son importantes para su vida Si $\square$ No $\square$

- Ha disminuido su interés sexual $\mathrm{Si} \square \mathrm{No} \square$

- Piensa que su enfermedad influye en la cantidad o calidad de sus relaciones $\mathrm{Si} \square \mathrm{No} \square$

- Lo relaciona con: la edad $\square$ el tiempo de convivencia con la pareja $\square$ la enfermedad $\square$ la medicación $\square$ Tto. no medicamentoso (por ejem aparatos) $\square$ dolor $\square$ fatiga $\square$ miedo $\square$

- Considera que las relaciones sexuales son igual de importantes para el hombre que para la mujer $\mathrm{Si} \square \mathrm{No} \square$

- Ha tenido relaciones con personas que no fueran pareja estable $\mathrm{Si} \square \mathrm{No} \square$ En caso afirmativo qué tipo de medidas de barrera ha utilizado 